\title{
Nonspecific Mouse Hepatitis Virus Positivity of Genetically Engineered Mice Determined by ELISA
}

\author{
Dae Jong Han ${ }^{1,2}$, Hyuncheol Kim ${ }^{2,3}$ and Su-Cheong Yeom ${ }^{4, \dagger}$ \\ ${ }^{I}$ Department of Research Laboratory, Biomedical Research Institute, \\ Seoul National University Hospital, Seoul 110-744, Korea \\ ${ }^{2}$ Interdisciplinary Program of Integrated Biotechnology, Sogang University, Seoul 121-742, Korea \\ ${ }^{3}$ Department of Chemical and Biomolecular Engineering, Sogang University, Seoul 121-742, Korea \\ ${ }^{4}$ Design Animal Resource Center, Pyeongchang Campus, Seoul National University, Kwangwon-do 232-916, Korea
}

\begin{abstract}
Mouse hepatitis virus (MHV) is a major pathogen in laboratory mice that usually leads to fatal diseases, such as hepatitis, multiple sclerosis, encephalitis, and respiratory disease. MHV has a high infection rate, and it needs to be detected as soon as possible to prevent its spread to other facilities. However, MHV detection by enzyme-linked immunosorbent assay (ELISA) often gives false positives; thus, it is very important that the results are confirmed as true positives in the early infection stage or distinguished as false positives with more accurate, reliable methods. Under microbiological screening, MHV ELISA-positive mice were found in four GFP-tagging transgenic mice. To verify the detection of the MHV antigen directly, reverse transcription polymerase chain reaction (RT-PCR) was performed, and the mice were determined to be MHV negative. Additional serum antibody-based screening was conducted with three different ELISA kits, and multiplexed fluorometric immunoassay (MFIA) was performed to confirm their accuracy/sensitivity. In brief, the ELISA kit for A59 nucleocapsid protein (MHV-A59N) revealed MHV ELISA positivity, while other ELISA kits (MHV-S lysate and MHV-JHM lysate) demonstrated MHV negativity. In MFIA, only the test for the recombinant A59 nucleocapsid antigen was MHV positive, which was consistent with the ELISA results. These results suggest that the ELISA kit with the recombinant A59 nucleocapsid antigen might induce non-specific MHV ELISA positivity and that confirmation is therefore essential.
\end{abstract}

Key Words: MHV, ELISA, RT-PCR, MFIA, IFA

\section{INTRODUCTION}

Mouse hepatitis virus (MHV) is a single-strand RNA coronavirus related to murine illnesses with high mortality. MHV is highly contagious, and it can be transmitted through aerosols and by direct contact (Wege et al., 1982). There

\footnotetext{
* Received: September 24, 2014 / Revised: December 4, 2014

Accepted: December 5, 2014

${ }^{\dagger}$ Corresponding author: Su-Cheong Yeom. Design Animal Resource Center, Pyeongchang Campus, Seoul National University, 1200 Shin-Ri, DaehwaMyeon, Pyeongchang-Gun, Kwangwon-do 232-916, Korea.

Tel: +82-33-339-5750, Fax: +82-33-339-5762

e-mail: scyeom@snu.ac.kr

C The Korean Society for Biomedical Laboratory Sciences. All rights reserved.
}

are two forms of tropism in mouse infections: polytropism (MHV-1, -2, -3, JHM and -A59), which is contracted via the respiratory system, and enterotropism (MHV-D, -Y, -RI, -S/CDC, LIVIM, and DVIM), which is more common and leads to infection in the intestine and excretion in feces (Cajal et al., 1962; Barthold et al., 1993; Rose and Weiss, 2009). MHV infection causes serious microbiological problems, and many research facilities and researchers are affected by MHV contamination. MHV infectivity depends on the age, genotype, health status, and experimental environment of the mouse. The primary infection causes hepatitis, encephalomyelitis, enteritis, and progressive wasting syndrome (Matthews et al., 2001; Eriksson et al., 2008). 
There are various methods for the detection of MHV infection; enzyme-linked immunosorbent assay (ELISA) can be easily performed with mouse serum samples in local laboratories. Reverse transcriptional polymerase chain reaction (RT-PCR) can also be used for detecting MHV virus antigen in feces and tissue samples. ELISA has been used as a routine screening method (Kagiyama et al., 1990) and there are many commercial MHV ELISA kits. However, each manufacturer uses specific MHV antigens, such as recombinant MHV-A59 strain nucleocapsid protein (MHVA59N) antigen, MHV-S supernatant, and MHV-JHM lysate. MHV isolation and histological diagnosis are direct detection methods, but they are difficult to perform in typical laboratories. RT-PCR may be a good method for the detection of MHV viral RNA (Yamada et al., 1998; Wang et al., 1999), but it also has limitations when the virus is cleared by the antibodies. Other MHV detection methods include indirect fluorescent assay (IFA), immuno-comb, and multiplexed fluorometric immunoassay (MFIA).

Severe B-cell-deficient mice are easily infected by MHV because of their impaired immune systems (Matthews et al., 2001; Matthews et al., 2002). Genetically engineered mice (GEMs) with immunodeficiency are also susceptible to viral infections (Lavi et al., 1995; Miura et al., 2008). In this study, we report an MHV false-positive ELISA result induced in a specific transgenic mouse breed.

\section{MATERIALS AND METHODS}

\section{Sample preparation}

In our institution, routine microbiological monitoring programs are performed every three months in which laboratory animals are routinely examined for 10 types of viruses and 17 pathogenic bacteria. During one such microbiological monitoring session, one of the sentinel mice from a quarantine room was determined to be MHV ELISA positive, and expanded monitoring was conducted to check for any MHV spread across the specific-pathogen free (SPF) barrier. Fresh blood samples from the orbital sinus and feces were collected from every mouse; 10 serum samples of the same volume were pooled into one vial, and it was verified that it showed a similar OD ratio. Feces from the same cage were pooled into one vial, and viral RNA extraction was conducted. If an MHV-positive ELISA result was detected, an additional ELISA test was conducted with the original individual serum samples, and RT-PCR was conducted. This study protocol was approved by our institution's Institutional Animal Care and Use Committee (IACUC).

\section{Antibody-based diagnosis via ELISA, IFA and MFI}

Three kinds of commercial MHV ELISA kits that can detect MHV antibodies of recombinant MHV-A59 nucleocapsid, S-strain supernatant, and JHM viral lysate were used. All ELISA experiments were conducted according to the manufacturer's instructions. For the IFA assay, $10 \mu \mathrm{l}$ of 1:2 diluted sera was loaded into the well of an MHV antigen-coated slide. The slide was incubated in a $37^{\circ} \mathrm{C}$ humidified incubator chamber for 40 minutes, and then each well was washed twice with $1 \mathrm{X}$ phosphate-buffered saline (PBS), and $10 \mu \mathrm{l}$ anti-mouse FITC conjugate was loaded into each well. After another 40 minutes of incubation and washing, each well was visualized using a 100X fluorescent microscope (Leica BM5500B, Mannheim, Germany). Three different antigen-specific MFIA panels (recombinant A59 nucleocapsid, A59 viral lysate, and S-strain viral lysate) were used, and tests were conducted by the Charles River Research Animal Diagnostic Service Center.

\section{Antigen-based diagnosis with RT-PCR}

Mouse feces were collected and mixed with $400 \mu \mathrm{l} 1 \mathrm{X}$ PBS. After vortexing, the supernatants were collected in clean tubes, and total RNA was extracted using the RNAiso solution (TaKaRa-Bio, Shiga, Japan) extraction method. The first-strand cDNAs were generated using EcoDry Premix RT kits (Clontech laboratories, Mountain View, CA). RTPCR was conducted as previously reported with specific primers (5'-GAAGTAGATAATGTAAGCGT-3' for forward and 5'-TTACACATTAGAGTCATCTTC-3' for reverse regions). A PCR kit (TaKaRa-Bio, Shiga, Japan) was used for MHV-PCR, and PCR was performed using a T3000 Thermal Cycler (Biometra, Goettingen, Germany). PCR amplicons were analyzed via $1.5 \%$ agarose gel electrophoresis and visualized using a UV trans-illuminator (BioRad, CA, USA). 
Table 1. Comparison of ELISA results from three different MHV ELISA kits

\begin{tabular}{|c|c|c|c|c|c|c|c|}
\hline \multirow{2}{*}{ No } & \multirow{2}{*}{ Strain } & \multicolumn{2}{|c|}{$\begin{array}{c}\text { A kit } \\
\left({ }^{\mathrm{a}} \mathrm{A} 59 \mathrm{~N}\right)\end{array}$} & \multicolumn{2}{|c|}{$\begin{array}{c}\text { B kit } \\
\text { (S viral lysate) }\end{array}$} & \multicolumn{2}{|c|}{$\begin{array}{c}\text { C kit } \\
\text { (JHM-strain) }\end{array}$} \\
\hline & & Results & OD & Results & OD & Results & OD \\
\hline P.C. & & $\mathrm{P}$ & $1.375^{b}$ & $\mathrm{P}$ & 0.568 & $\mathrm{P}$ & 1.195 \\
\hline 1 & B6.GFP-Foxp3 KI & $\mathrm{E}$ & 0.148 & $\mathrm{~N}$ & 0.002 & $\mathrm{~N}$ & 0.096 \\
\hline 2 & B6.GFP-Foxp3 *CD45.1 & $\mathrm{P}$ & 0.551 & $\mathrm{~N}$ & 0.005 & $\mathrm{~N}$ & 0.117 \\
\hline 3 & B6.GFP-Foxp3 *CD45.1 & $\mathrm{P}$ & 1.458 & $\mathrm{~N}$ & 0.005 & $\mathrm{~N}$ & 0.093 \\
\hline 4 & B6.GFP-Foxp3 KI & $\mathrm{P}$ & 0.913 & $\mathrm{~N}$ & 0.04 & $\mathrm{~N}$ & 0.233 \\
\hline 5 & Strain A (positive) & $\mathrm{P}$ & 1.003 & $\mathrm{~N}$ & 0.454 & $P$ & 1.22 \\
\hline 6 & Strain B (negative) & $\mathrm{N}$ & 0.002 & $\mathrm{~N}$ & 0.002 & $\mathrm{~N}$ & 0.008 \\
\hline
\end{tabular}

Optical density (OD) ratio of positive samples (A kit: $>0.39$, B kit: $>0.568, \mathrm{C}$ kit: $>0.3$ )

${ }^{a}$ A59N: recombinant A59 nucleocapsid

${ }^{\mathrm{b}}$ Bold numbers indicate positive results from each ELISA kit

\section{Direct transmission test with C57BL/6 and NOD/ SCID.IL-2rg ${ }^{-1-}$}

Four B6.GFP-FoxP3 knock-in mice were selected, and direct transmission tests were conducted. The mice were separated into different cages, and they were cohoused with C57BL/6 and NOD/SCID.IL-2 rg $^{-/-}$(NSG) mice (1:1). After two and four weeks of cohousing, the C57BL/6, NSG, and B6.GFP-FoxP3 knock-in mice were necropsied and tested for MHV.

\section{Statistical analysis}

Statistical analysis was conducted using SPSS 20 (SPSS Inc., Chicago, IL) for Pearson correlation analysis, and $P<0.05$ was regarded as significant.

\section{RESULTS}

In the MHV screening test for quarantine, one of the 17 samples was MHV ELISA positive. Additional MHV screening was conducted for 86 mice with the MHVA59N-coated ELISA kit, and 16 mice were found to be MHV ELISA positive. To confirm MHV infection, RT-PCR was conducted, and eight mice were MHV RT-PCR positive according to RT-PCR. We sought to verify the MHV spread across the SPF barrier. In the MHV ELISA screening test, four pooled serum samples showed high optical density.
The original 40 serum samples from these pools were tested again, and four serum samples had high OD ratios. In particular, when tested with an ELISA A kit, MHV ELISA-positive serums were focused on the B6.GFP-Foxp3 knock-in mice (Table 1). To confirm MHV with viral RNA detection, RT-PCR was performed, but the results were not consistent with those of the MHV-A59N ELISA. Only one sample (Sample 5) from the quarantine room was MHV RT-PCR positive (Table 2). There were three possible explanations for this inconsistency: 1) There was a non-specific positive MHV-A59N ELISA result, 2) the mice had already eliminated the virus via their immune response, or 3) a new type of MHV infection had been detected. Additional antibody detection tests were conducted with two different MHV ELISA B and C kits (S lysate and JHM lysate) (Table 1), and the IFA method was used to explore these possibilities (Table 2). One positive control and one serum sample (Sample 5) from the quarantine room were ELISA and IFA positive, but no FoxP3 knock-in serum samples (Samples 1 4) were ELISA positive when tested with the ELISA B and $\mathrm{C}$ kits. This indicated that the ELISA results from the MHV-A59N antigen might be non-specifically positive.

To confirm the non-specific MHV positivity of the A59N antigen, an MFIA analysis was performed with three different antigens (A59N, A59 lysate, and S lysate) at the Charles River Research Animal Diagnostic Service Center (MA, 
Table 2. Indirect fluorescence assay (IFA), RT-PCR, and multiplexed fluorometric immunoassay (MFIA) for MHV confirmation

\begin{tabular}{|c|c|c|c|c|c|c|}
\hline \multirow{2}{*}{ No } & \multirow{2}{*}{ Strain } & \multirow{2}{*}{ IFA } & \multirow{2}{*}{ RT-PCR } & MFIA & MFIA & MFIA \\
\hline & & & & $(\mathrm{A} 59 \mathrm{~N})$ & (A59 lysate) & (S lysate) \\
\hline 1 & B6.GFP-Foxp3 KI & $\mathrm{N}$ & $\mathrm{N}$ & 2 & - & - \\
\hline 2 & B6.GFP-Foxp3 ${ }^{*}$ CD45.1 & $\mathrm{N}$ & $\mathrm{N}$ & 3 & - & - \\
\hline 3 & B6.GFP-Foxp3 $*$ CD 45.1 & $\mathrm{~N}$ & $\mathrm{~N}$ & 11 & $3^{\mathrm{a}}$ & - \\
\hline 4 & B6.GFP-Foxp3 KI & $\mathrm{N}$ & $\mathrm{N}$ & 6 & - & - \\
\hline 5 & Strain A (positive) & $\mathrm{P}$ & $\mathrm{P}$ & 23 & 21 & 11 \\
\hline 6 & Strain B (negative) & $\mathrm{N}$ & $\mathrm{N}$ & - & - & - \\
\hline
\end{tabular}

${ }^{\mathrm{a}} \mathrm{A}$ net score of 3 indicates positivity to MHV A59 lysate, but this was confirmed to be negative via IFA.

Table 3. Correlation analysis between MFI and ELISA kit A

\begin{tabular}{llcc}
\hline \hline & $\begin{array}{c}\text { MFI } \\
\text { (A59N) }\end{array}$ & $\begin{array}{c}\text { MFI } \\
\text { (A59 lysate) }\end{array}$ & $\begin{array}{c}\text { MFI } \\
\text { (S lysate) }\end{array}$ \\
\hline Pearson correlation & 0.900 & 0.821 & 0.400 \\
$P$ value & $0.037^{\mathrm{a}}$ & 0.890 & 0.505 \\
$\mathrm{n}$ & 5 & 5 & 5 \\
\hline
\end{tabular}

${ }^{a}$ Significant correlation

USA), and the results were consistent with conventional ELISA. MHV-positive sera showed high values for all antigens, but B6.GRP-FoxP3 knock-in sera were only significant in MFIA with the A59N antigen. When MFIA was performed with the MHV-A59N antigen, three of the B6.GFP-Foxp3-tagging transgenic mice (Samples 2 4) were positive, and the other (Sample 1) was equivocal, consistent with the results of the ELISA A kit. All B6.GFPFoxp3-tagging transgenic mice (Samples 1 4) were negative when tested with the other two viral antigens (Table 2). The correlation between the ELISA A and MFIA results with the same recombinant antigen was remarkably high, because each serological kit was coated with the same recombinant A59N antigens (Table 3). To confirm the results of Sample no. 1, MHV IFA was performed, and the four samples produced no clearly visible fluorescent spot. Additionally, Sample no. 3 appeared to be positive when tested with A59 viral lysate MFIA, but it was finally confirmed to be negative with MHV IFA (Fig. 1A). RT-PCR found that only Sample no. 5 Strain A was positive (Fig. 1B). Additional statistical analysis also indicated that the

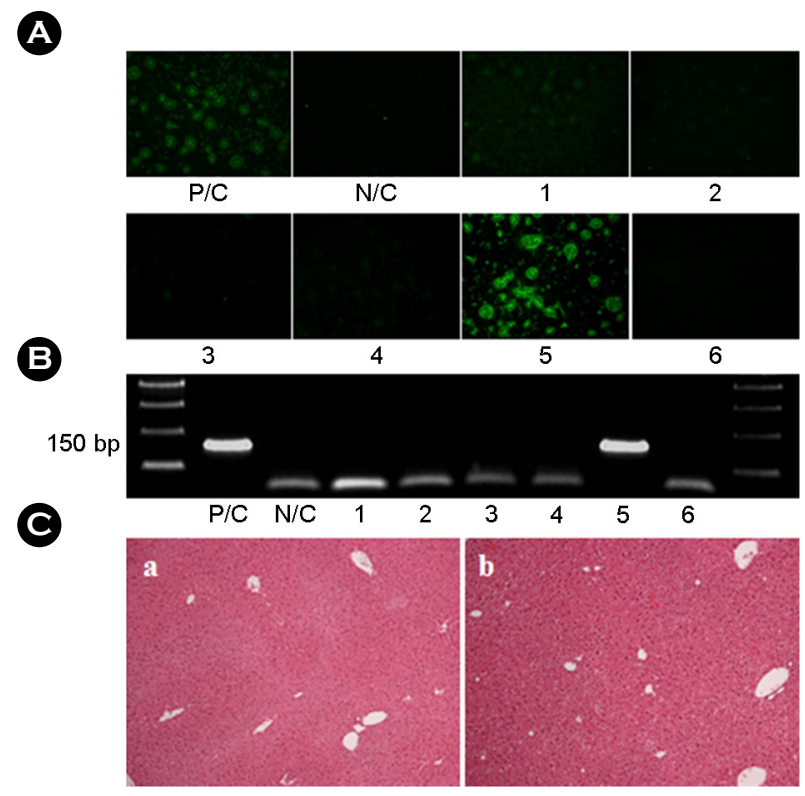

Fig. 1. MHV screening with indirect fluorescent assay (IFA), RT-PCR and histological examination. (A) The positive control and sample no. 5 were determined to be positive, the same as previous serological tests. Samples no. 1-4, and 6 did not exhibit any clear fluorescence spot and were negative. (B) MHV RT-PCR. All samples were tested with fecal RT-PCR to detect MHV antigens. (C) Histological examination was performed on the liver tissues of the MHV non-specific positive mouse (a) and negative mouse (b) (X100). P/C: positive control, N/C: negative control, 1-6: serum samples.

A59N antigen was correlated with non-specific MHVpositive results. It was determined that Strain A (Sample 5) was really infected with MHV, because three ELISA methods (IFA, RT-PCR, and MFIA) all indicated positive. However, B6.GFP-Foxp3 mouse serum samples (Samples 1 4) only 
reacted with the recombinant A59N MHV antigens.

It was also important to find liver necrosis when they were really infected with MHV, although the MHV antibodies were already eliminated. The livers of the B6.GFP-Foxp3 mice did not show any pathological changes upon histological examination (Fig. 1C). The possibility of a new murine virus was evaluated using a contact-rearing test. It was assumed that if a new murine virus had infected the B6.GFP-FoxP3 knock-in mice, it would be transmissible. However, no B6 or NSG mice co-reared with B6.GFPFoxP3 knock-in mice were positive for MHV on ELISA or RT-PCR (Fig. 1B).

\section{DISCUSSION}

MHV is a common viral disease in laboratory mice for which they should be regularly screened. Serological methods such as ELISA are commonly used to detect viruses in many experimental animal laboratories and holding facilities. ELISA might be an inexpensive and simple monitoring method, but it can lead to false positives, false negatives, and misinterpretation. Thus, other methods such as IFA and/or RT-PCR are typically used for confirmation. In this study, non-specific MHV ELISA results were detected, particularly in the B6.GFP-FoxP3 knock-in strain. Non-specific positive results were only detected in ELISA kits with the A59N antigen, and this was confirmed by MFIA analysis and correlation analysis. This non-specific result differed from false-negative or false-positive results. In addition, there was no transmission between positive B6.GFP-FoxP3 knock-in mice and co-reared B6 and NSG mice, so these non-specific ELISA results could not have been caused by a new type of MHV. The B6.GFP-FoxP3 knock-in strain was not MHV ELISA positive in a previous examination with an ELISA kit using an A59N antigen. It is unclear what molecule induced the non-specific MHV ELISA results associated with B6.GFP-Foxp3 transgenic mice sera binding to the recombinant MHV-A59 strain nucleocapsid protein coated on the ELISA A kits and MFIA panels.

Sometimes, widely used GFP-tagging transgenic animals may be affected by certain side effects of renal pathology, such as glomerulosclerosis (Guo et al., 2007). It seems that transgenic mice may produce certain non-specific proteins such that serological false-positive results are occasionally detected. Thus, this sometimes occurs in ELISA, and the results are easily misinterpreted as positive at the first serological MHV screening. Confirmation with another serological test kit is recommended, and other applicable methods for diagnosis are necessary, because initial treatments are most important in preventing the spread of MHV. In conclusion, it was confirmed that non-specific MHV ELISA positivity can occur in GEMs. Although a recombinant protein caused the non-specific response in this study, serological methods might induce similar results; thus, confirmation is necessary. Finally, this is the first report of non-specific MHV positivity in ELISA involving GEMs.

\section{Acknowledgements}

This work was supported by the Ministry of Food and Drug Safety (KFDA2013-18075).

\section{REFERENCES}

Barthold SW, Beck DS, Smith AL. Enterotropic coronavirus (mouse hepatitis virus) in mice: influence of host age and strain on infection and disease. Lab Anim Sci. 1993. 43: 276 -284 .

Cajal N, Burducea O, Popescu G. Experimental investigations of murine hepatitis. I. Evolution of hepatitis induced with MHV-2 andMHV-4 viruses in mice exposed to the action of radiophosphorus (P-32) and radioiodine (I-131). Stud Cercet Inframicrobiol. 1962. 13: 163-167.

Eriksson KK, Cervantes-Barragan L, Ludewig B, Thiel V. Mouse hepatitis virus liver pathology is dependent on ADP-ribose-1"phosphatase, a viral function conserved in the alpha-like supergroup. J Virol. 2008. 82: 12325-12334.

Guo JK, Cheng EC, Wang L, Swenson ES, Ardito TA, Kashgarian M, Cantley LG, Krause DS. The commonly used beta-actinGFP transgenic mouse strain develops a distinct type of glomerulosclerosis. Transgenic Res. 2007. 16: 829-834.

Kagiyama N, Takakura A, Terada E, Sakurai Y, Suzuki H. Studies on the development of an ELISA kit for microbiological monitoring. 2. Improvement of the prototype ELISA kit with 
special references to mouse hepatitis virus antigen. Jikken Dobutsu. 1990. 39: 97-102.

Lavi E, Wang Q, Gombold J, Sutherland R, Paterson Y, Weiss S. Pathology of MHV-A59 infection in beta 2 microglobulin negative mice. Adv Exp Med Biol. 1995. 380: 179-181.

Matthews AE, Weiss SR, Lavi E, Shlomchik M, Paterson Y. The role of $\mathrm{B}$ cells in mouse hepatitis virus infection and pathology. Adv Exp Med Biol. 2001. 494: 363-368.

Matthews AE, Lavi E, Weiss SR, Paterson Y. Neither B cells nor $\mathrm{T}$ cells are required for CNS demyelination in mice persistently infected with MHV-A59. J Neurovirol. 2002. 8: 257 -264 .

Miura TA, Travanty EA, Oko L, Bielefeldt-Ohmann H, Weiss $\mathrm{SR}$, Beauchemin N, Holmes KV. The spike glycoprotein of murine coronavirus MHV-JHM mediates receptor-independent infection and spread in the central nervous systems of
Ceacam1a-/- Mice. J Virol. 2008. 82: 755-763.

Rose KM, Weiss SR. Murine coronavirus cell type dependent interaction with the type I interferon response. Viruses. 2009. 1: 689-712.

Wang RF, Campbell WL, Cao WW, Colvert RM, Holland MA, Cerniglia CE. Diagnosis of mouse hepatitis virus contamination in mouse population by using nude mice and RT-PCR. Mol Cell Probes. 1999. 13: 29-33.

Wege H, Siddell S, ter Meulen V. The biology and pathogenesis of coronaviruses. Curr Top Microbiol Immunol. 1982. 99: 165 -200 .

Yamada YK, Yabe M, Takimoto K, Nakayama K, Saitoh M. Application of nested polymerase chain reaction to detection of mouse hepatitis virus in fecal specimens during a natural outbreak in an immunodeficient mouse colony. Exp Anim. 1998. 47: 261-264. 